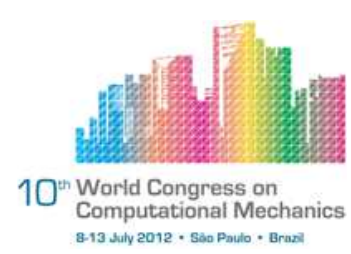

\title{
3D VS. 2D MODELLING OF CRACKING AND PLASTICITY IN POLYCRYSTALLINE MATERIALS
}

\author{
M. Paggi ${ }^{1}$, E. Lehmann ${ }^{2}$, C. Weber ${ }^{2}$, A. Carpinteri ${ }^{1}$, P. Wriggers ${ }^{2}$ \\ ${ }^{1}$ Department of Structural, Geotechnical and Building Engineering, Politecnico di Torino, \\ Torino, Italy (marco.paggi@polito.it) \\ ${ }^{2}$ Institute of Continuum Mechanics, Leibniz University of Hannover, Hannover, Germany
}

\begin{abstract}
The problem of cracking and plasticity in face-centered-cubic polycrystals is herein investigated. The aim is to quantify the difference between $3 D$ simulations, computationally expensive but able to model real polycrystalline geometries, and simplified computations on $2 D$ cross-sections with a reduced computational cost. To this aim, a unified finite element formulation with elasto-plastic elements for the grain interior and interface elements for the grain boundaries is developed. This approach is suitable for the analysis of polycrystalline materials with a response intermediate between that of brittle ceramics and that of ductile metals. Crystal plasticity theory is used for 3D computations, whereas isotropic VON MISES plasticity is adopted for the 2D tests. For the grain boundaries, a cohesive zone model (CZM) accounting for Mode Mixity is considered. Examining the nonlinearity due to the CZM only, $3 D$ simulations of uniaxial tensile tests differ from $2 D$ predictions due to the higher tortuosity of the crack path in 3D, leading to significant Mixed Mode deformation. Regarding the comparison between $3 D$ and $2 D$ simulations with plasticity only, results show that the saturation of the stress field is much faster in $3 D$ than in $2 D$. Finally, when both nonlinearities are simultaneously present, a strong interplay is evidenced. The CZM prevails over plasticity for low deformation levels. Afterwards, plasticity prevails over CZM. Finally, for a very large deformation, failure is ruled by the CZM formulation which induces a softening in the global structural response and a size-scale dependency of the results.
\end{abstract}

Keywords: Cohesive Zone Model; Crystal plasticity; Isotropic plasticity; Finite element method; Polycrystalline materials.

\section{INTRODUCTION}

At present, in spite of the inherent 3D geometry of polycrystalline materials, most of the numerical investigations based on the finite element method are still restricted to 2D topologies [1-6]. This is mainly due to the higher computational cost required to solve a nonlinear problem in $3 \mathrm{D}$, to mesh complex geometries and to efficiently post-process the numerical results. So far, the limitation of using 2D models over more realistic 3D simulations has not 
yet been fully quantified, although it is an information of primary importance from the engineering point of view. In case of intergranular crack growth, for instance, only qualitative statements regarding the crack pattern, much more tortuous in $3 \mathrm{D}$ than in $2 \mathrm{D}$, are available [7].

In the present study, 3D and 2D uniaxial tensile tests of a face-centered-cubic polycrystal microstructure are simulated. The aim is to better quantify the difference between 3D and 2D simulations in terms of structural predictions. Plasticity inside the grains and grain boundary decohesion are both considered, in order to propose a unified model able to deal with the main forms of nonlinearity of a polycrystalline material. Experimental tests show in fact both the occurrence of plastic deformation in ductile metals, and intergranular and transgranular cracking in ceramic polycrystals. Actually, it is not possible to exclude intermediate situations in materials science where the grains are relatively ductile and the interfaces quite brittle. This is for instance the case when chemical segregation of a brittle component takes place along the grain boundaries of a ductile metal polycrystal [8]. In these situations, a mechanically coupled model is expected to be useful.

Hence, to quantify the interplay between grain boundary decohesion and grain plasticity, the cohesive zone model (CZM) and the theory of crystal plasticity are implemented in the finite element program FEAP [9]. Their main features are summarized in Sections 2 and 3, respectively. Crystal plasticity is effective for the study of ductile heterogeneous materials, like metals used in rolling and forming operations. On the other hand, the CZM can be efficiently used to model localized cracking at grain boundaries typical of brittle materials. In Section 3, the method used to generate the finite element meshes is described. In Sections 4 and 5, virtual tensile tests on 3D polycrystalline material microstructures are performed and the results are compared with those obtained from analogous simulations considering 2D cross-sections under the assumption of plane strain deformation. Finally, the interplay between plasticity and CZM depending on the parameters of the elementary models is investigated in Section 6. Concluding remarks complete the article.

\section{CONSTITUTIVE RELATION FOR THE GRAIN BOUNDARIES: THE COHESIVE ZONE MODEL}

Grain boundaries are often considered as perfectly bonded interfaces. However, these material discontinuities are often the source of damage and cracking in polycrystalline materials and imperfect bonding is a more realistic assumption [5,10]. To this aim, interface elements for 3D nonlinear crack propagation problems have been implemented in the finite element program FEAP [9] by the present authors. The interface element is a finite element with zero-thickness that is used to impose tractions-relative displacement constitutive relations for the grain boundaries. In the kinematic part of the interface element, the relative opening and sliding displacements of the opposing nodes sharing a common interface are computed. The cohesive tractions are then computed in terms of these relative opening and sliding displacements according to the specified CZM, see also [11-13]. In the present study, the CZM by Tvergaard [14] is adopted, since it is frequently used to model interface crack propagation along pre-existing interfaces. In 3D, a local reference system defined by the normal vector $\mathbf{n}$ and two orthogonal tangent vectors $\mathbf{t}_{1}$ and $\mathbf{t}_{\mathbf{2}}$ in the plane of the interface element is introduced. The normal and tangential tractions, $\sigma, \tau_{1}$ and $\tau_{2}$ are computed as functions of the 
normal and tangential relative displacements $g_{\mathrm{N}}, g_{\mathrm{T}, 1}$ and $g_{\mathrm{T}, 2}$ :

$$
\begin{aligned}
\sigma & =\sigma_{\mathrm{p}} \frac{g_{\mathrm{N}}}{l_{\mathrm{Nc}}} P(\lambda), \\
\tau_{1} & =\gamma \sigma_{\mathrm{p}} \frac{g_{\mathrm{T}, 1}}{l_{\mathrm{Tc}}} P(\lambda), \\
\tau_{2} & =\gamma \sigma_{\mathrm{p}} \frac{g_{\mathrm{T}, 2}}{l_{\mathrm{Tc}}} P(\lambda),
\end{aligned}
$$

where

$$
\begin{aligned}
P(\lambda) & = \begin{cases}\frac{27}{4}\left(1-2 \lambda+\lambda^{2}\right), & \text { for } 0 \leq \lambda \leq 1 \\
0, & \text { otherwise }\end{cases} \\
\lambda & =\sqrt{\left(\frac{g_{\mathrm{N}}}{l_{\mathrm{Nc}}}\right)^{2}+\left(\frac{g_{\mathrm{T}, 1}}{l_{\mathrm{Tc}}}\right)^{2}+\left(\frac{g_{\mathrm{T}, 2}}{l_{\mathrm{Tc}}}\right)^{2}}
\end{aligned}
$$

The normal and resultant tangential tractions are shown in Fig. 1 vs. $g_{\mathrm{N}}$ and $g_{\mathrm{T}}=\sqrt{g_{\mathrm{T}, 1}^{2}+g_{\mathrm{T}, 2}^{2}}$. In this formulation, $l_{\mathrm{Nc}}$ and $l_{\mathrm{Tc}}$ are critical separations in the normal and tangential directions corresponding to vanishing cohesive tractions. The parameter $\sigma_{\mathrm{p}}$ defines the Mode I peak CZM traction, whereas the Mode II peak CZM traction is $\tau_{\mathrm{p}}=\gamma \sigma_{\mathrm{p}}$. The variable $\lambda$ physically represents a measure of the total relative displacement experienced by the interface.

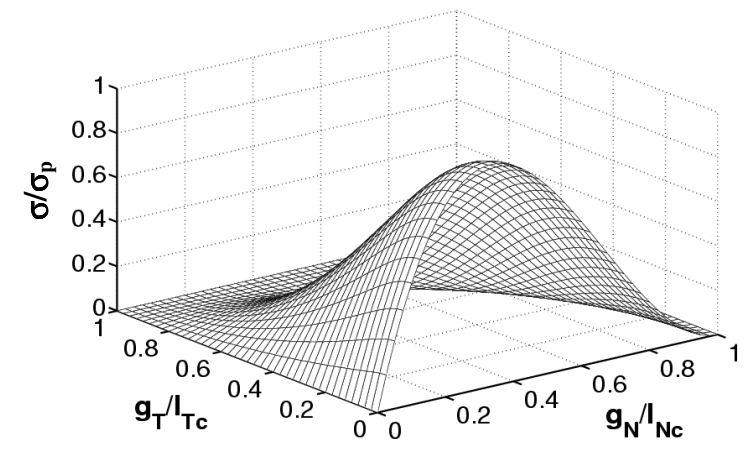

(a) Normal cohesive tractions

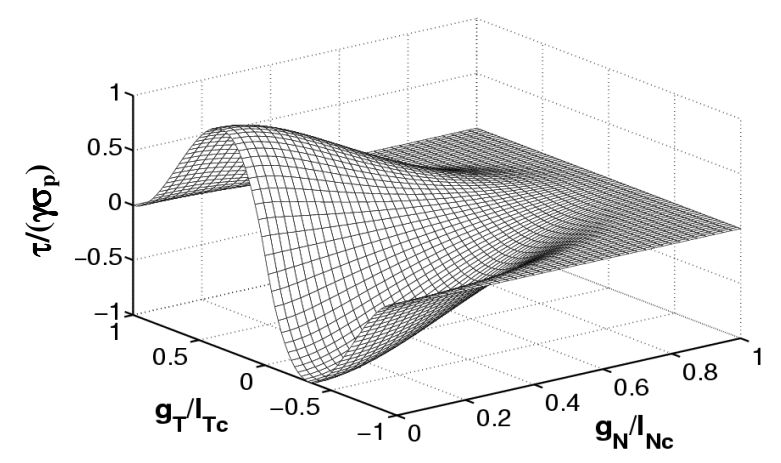

(b) Tangential cohesive tractions

Figure 1. Normal and tangential cohesive tractions.

The implementation of this element has been carried out in FEAP [9] by following the methodology described in [5, 15-17] and considering different element topologies, e.g., interface elements connecting linear or quadratic tetrahedral elements and linear or quadratic brick elements. Due to the nonlinearity of the CZM and the analysis carried out in an implicit FEM code, the consistent linearization of the weak form of the element is performed to compute its tangent stiffness matrix and the residual to be used in a NEWTON-RAPHSON iterative procedure. To this aim, the partial derivatives of the cohesive tractions with respect to the normal and tangential relative displacements are used to determine the tangent constitutive matrix of 
the interface element [5]. For the CZM in Eq.(1) the derivatives are:

$$
\begin{aligned}
\frac{\partial \sigma}{\partial g_{\mathrm{N}}} & =\sigma_{\mathrm{p}} \frac{P}{l_{\mathrm{Nc}}}+\sigma_{\mathrm{p}} \frac{g_{\mathrm{N}}}{l_{\mathrm{Nc}}} \frac{\partial P}{\partial \lambda} \frac{\partial \lambda}{\partial g_{\mathrm{N}}}, \\
\frac{\partial \sigma}{\partial g_{\mathrm{T}, 1}} & =\sigma_{\mathrm{p}} \frac{g_{\mathrm{N}}}{l_{\mathrm{Nc}}} \frac{\partial P}{\partial \lambda} \frac{\partial \lambda}{\partial g_{\mathrm{T}, 1}}, \\
\frac{\partial \sigma}{\partial g_{\mathrm{T}, 2}} & =\sigma_{\mathrm{p}} \frac{g_{\mathrm{N}}}{l_{\mathrm{Nc}}} \frac{\partial P}{\partial \lambda} \frac{\partial \lambda}{\partial g_{\mathrm{T}, 2}}, \\
\frac{\partial \tau_{1}}{\partial g_{\mathrm{N}}} & =\gamma \sigma_{\mathrm{p}} \frac{g_{\mathrm{T}, 1}}{l_{\mathrm{Tc}}} \frac{\partial P}{\partial \lambda} \frac{\partial \lambda}{\partial g_{\mathrm{N}}}, \\
\frac{\partial \tau_{1}}{\partial g_{\mathrm{T}, 1}} & =\gamma \sigma_{\mathrm{p}} \frac{P}{l_{\mathrm{Tc}}}+\gamma \sigma_{\mathrm{p}} \frac{g_{\mathrm{T}, 1}}{l_{\mathrm{Tc}}} \frac{\partial P}{\partial \lambda} \frac{\partial \lambda}{\partial g_{\mathrm{T}, 1}}, \\
\frac{\partial \tau_{1}}{\partial g_{\mathrm{T}, 2}} & =\gamma \sigma_{\mathrm{p}} \frac{g_{\mathrm{T}, 1}}{l_{\mathrm{Tc}}} \frac{\partial P}{\partial \lambda} \frac{\partial \lambda}{\partial g_{\mathrm{T}, 2}}, \\
\frac{\partial \tau_{2}}{\partial g_{\mathrm{N}}} & =\gamma \sigma_{\mathrm{p}} \frac{g_{\mathrm{T}, 2}}{l_{\mathrm{Tc}}} \frac{\partial P}{\partial \lambda} \frac{\partial \lambda}{\partial g_{\mathrm{N}}}, \\
\frac{\partial \tau_{2}}{\partial g_{\mathrm{T}, 1}} & =\gamma \sigma_{\mathrm{p}} \frac{g_{\mathrm{T}, 2}}{l_{\mathrm{Tc}}} \frac{\partial P}{\partial \lambda} \frac{\partial \lambda}{\partial g_{\mathrm{T}, 1}}, \\
\frac{\partial \tau_{2}}{\partial g_{\mathrm{T}, 2}} & =\gamma \sigma_{\mathrm{p}} \frac{P}{l_{\mathrm{Tc}}}+\gamma \sigma_{\mathrm{p}} \frac{g_{\mathrm{T}, 2}}{l_{\mathrm{Tc}}} \frac{\partial P}{\partial \lambda} \frac{\partial \lambda}{\partial g_{\mathrm{T}, 2}} .
\end{aligned}
$$

\section{CONSTITUTIVE RELATION FOR THE GRAINS: MULTIPLICATIVE MULTI- SURFACE ELASTO-PLASTICITY}

A crystal plasticity formulation [18-20] is adopted to describe the constitutive behaviour of polycrystalline grains in 3D. The deformation gradient $\boldsymbol{F}=\frac{\partial \boldsymbol{x}}{\partial \boldsymbol{X}}$ with Jacobian $\mathrm{J}=\operatorname{det} \boldsymbol{F}>0$ maps tangent vectors of material lines in the reference configuration $\mathcal{B} \in \mathbb{R}^{3}$ onto tangent vectors of deformed lines in the current configuration $\mathcal{B}_{\mathrm{t}} \in \mathbb{R}^{3}$ and is decomposed into an elastic and a plastic part. The elastic part $\boldsymbol{F}^{e}$ contributes to stretching and rigid body rotation of the crystal lattice, the plastic part $\boldsymbol{F}^{p}$ characterises plastic flow caused by dislocations on defined slip systems

$$
\boldsymbol{F}=\boldsymbol{F}^{e} \boldsymbol{F}^{p}
$$

The multiplicative split assumes a local unstressed intermediate configuration defined by the plastic deformation gradient, see Fig. 2, which can be determined through an evolution eqaution and whose initial condition is assumed to be $\boldsymbol{F}_{0}^{p}=\mathbf{1}$.

Further, a volumetric-deviatoric split of the deformation gradient and its constituents is performed

$$
\boldsymbol{F}_{\text {iso }}=\mathbf{J}^{-1 / 3} \boldsymbol{F}, \quad \boldsymbol{F}_{\text {iso }}^{e}=\mathbf{J}^{e-1 / 3} \boldsymbol{F}^{e}, \quad \boldsymbol{F}_{\text {iso }}^{p}=\mathbf{J}^{p-1 / 3} \boldsymbol{F}^{p}
$$

with $\mathbf{J}=\mathbf{J}^{e}$ due to fulfilling the requirement of present plastic incompressibility expressed through $\mathrm{J}^{p}=1$. 


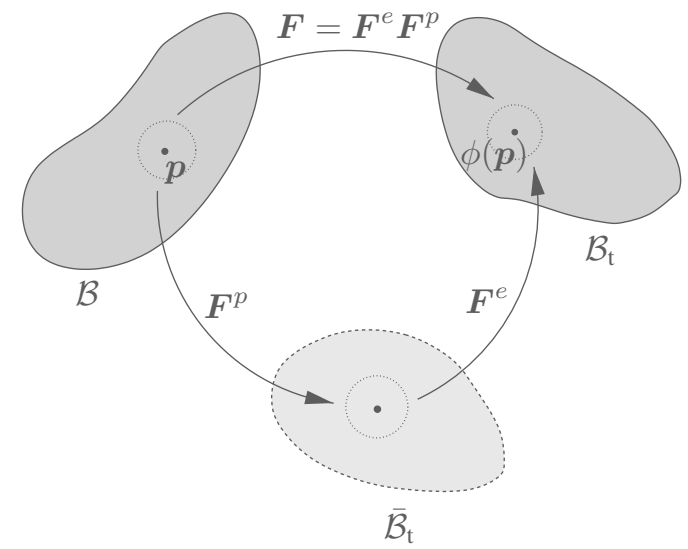

(a) Continuum mechanical framework

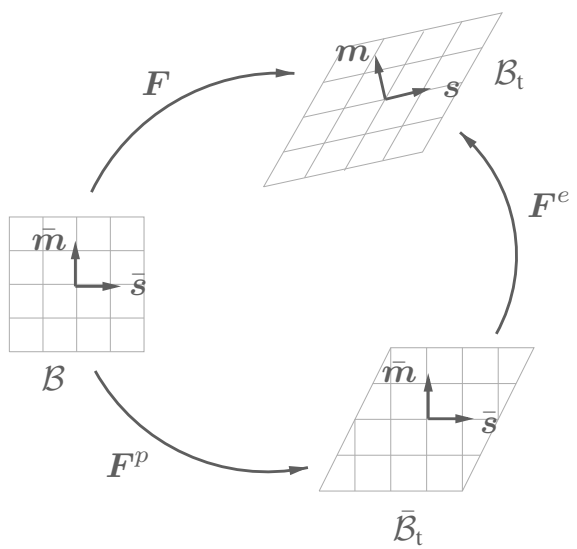

(b) Crystallographic concept

Figure 2. Multiplicative elasto-plastic decomposition of the deformation gradient $\boldsymbol{F}$.

\subsection{Thermodynamical considerations}

The deformation power per unit undeformed volume can be written as

$$
\boldsymbol{P}: \dot{\boldsymbol{F}}=\overline{\boldsymbol{P}}: \dot{\boldsymbol{F}}^{e}+\overline{\boldsymbol{\Sigma}}: \overline{\boldsymbol{L}}^{p}
$$

where $\overline{\boldsymbol{P}}=\boldsymbol{P} \boldsymbol{F}^{p T}$ is the $1^{\text {st }}$ PIOLA-KIRCHHOFF stress tensor relative to the intermediate configuration $\overline{\mathcal{B}}_{\mathrm{t}}$ and $\bar{\Sigma}=\boldsymbol{F}^{e T} \boldsymbol{P} \boldsymbol{F}^{p T}=\boldsymbol{F}^{e T} \boldsymbol{\tau} \boldsymbol{F}^{e-T}$ a stress measure conjugate to the plastic velocity gradient $\overline{\boldsymbol{L}}^{p}=\dot{\boldsymbol{F}}^{p} \boldsymbol{F}^{p-1}$ on $\overline{\mathcal{B}}_{\mathrm{t}}, \boldsymbol{\tau}$ being the KIRCHHOFF stress tensor on $\mathcal{B}_{\mathrm{t}}$. Further, it is

$$
\overline{\boldsymbol{P}}=\boldsymbol{F}^{e} \overline{\boldsymbol{S}}, \quad \overline{\boldsymbol{S}}=\overline{\boldsymbol{C}}^{e-1} \overline{\boldsymbol{\Sigma}}, \quad \overline{\boldsymbol{C}}^{e}=\boldsymbol{F}^{e T} \boldsymbol{F}^{e}
$$

where $\overline{\boldsymbol{S}}$ is the $2^{\text {nd }}$ PIOLA-KIRCHHOFF stress tensor relative to the intermediate configuration $\overline{\mathcal{B}}_{\mathrm{t}}$ which is symmetric, $\overline{\boldsymbol{C}}^{e}$ is further the elastic right CAUCHY-GREEN tensor on $\overline{\mathcal{B}}_{\mathrm{t}}$.

The evolution of the plastic deformation gradient $\boldsymbol{F}^{p}$ is defined by the plastic flow equation, resulting from the plastic rate of deformation $\overline{\boldsymbol{L}}^{p}$. In the presence of $n_{\text {syst }}$ systems undergoing plastic slip, represented by the plastic shear rates $\dot{\gamma}^{\alpha}$, the plastic flow equation is further generalised

$$
\overline{\boldsymbol{L}}^{p}=\dot{\boldsymbol{F}}^{p} \boldsymbol{F}^{p-1}, \quad \overline{\boldsymbol{L}}^{p}=\sum_{\alpha=1}^{n_{\mathrm{syst}}} \dot{\gamma}^{\alpha} \overline{\boldsymbol{s}}^{\alpha} \otimes \overline{\boldsymbol{m}}^{\alpha},
$$

$\overline{\boldsymbol{s}}^{\alpha}$ being the slip direction vector and $\overline{\boldsymbol{m}}^{\alpha}$ being the slip plane normal vector of the $\alpha$-th slip system $\left\{\overline{\boldsymbol{s}}^{\alpha}, \overline{\boldsymbol{m}}^{\alpha}\right\}$. The slip system vectors have the properties $\overline{\boldsymbol{s}} \cdot \overline{\boldsymbol{m}}=0$ and thus $\left(\overline{\boldsymbol{s}}^{\alpha} \otimes \overline{\boldsymbol{m}}^{\alpha}\right)\left(\overline{\boldsymbol{s}}^{\alpha} \otimes \overline{\boldsymbol{m}}^{\alpha}\right)=\mathbf{0}$. The generalisation in (8) leads to the modified evolution equation of the plastic deformation gradient depending on the plastic slips

$$
\dot{\boldsymbol{F}}^{p}=\left[\sum_{\alpha} \dot{\gamma}^{\alpha} \overline{\boldsymbol{s}}^{\alpha} \otimes \overline{\boldsymbol{m}}^{\alpha}\right] \boldsymbol{F}^{p} .
$$




\subsection{The resolved SCHMID stress}

The SCHMID stress $\tau^{\alpha}$ is the projection of $\overline{\boldsymbol{\Sigma}}$ onto the slip system $\overline{\boldsymbol{s}}^{\alpha} \otimes \overline{\boldsymbol{m}}^{\alpha}$

$$
\tau^{\alpha}=\left(\operatorname{dev}[\overline{\boldsymbol{\Sigma}}] \cdot \overline{\boldsymbol{m}}^{\alpha}\right) \cdot \overline{\boldsymbol{s}}^{\alpha}=\operatorname{dev}[\overline{\boldsymbol{\Sigma}}]: \overline{\boldsymbol{s}}^{\alpha} \otimes \overline{\boldsymbol{m}}^{\alpha} .
$$

As the slip system tensor $\overline{\boldsymbol{s}}^{\alpha} \otimes \overline{\boldsymbol{m}}^{\alpha}$ is purely deviatoric, only the deviator of the stress tensor contributes to the resolved stress. With the relations in (7) and some straightforward recast, it is

$$
\tau^{\alpha}=\boldsymbol{R}^{e T} \boldsymbol{\tau} \boldsymbol{R}^{e}: \overline{\boldsymbol{s}}^{\alpha} \otimes \overline{\boldsymbol{m}}^{\alpha}
$$

\subsection{Elastic response}

The elastic part of the deformation is gained from a NEO-HOOKEean strain energy function. Due to assumed isotropy within the elastic contribution, the description is given in terms of the elastic left CAUCHY-GREEN tensor $\boldsymbol{b}^{e}$. Applying a volumetric-deviatoric split yields

$$
\begin{gathered}
\rho \psi\left(\boldsymbol{b}_{\mathrm{iso}}^{e}, \mathbf{J}^{e}\right)=\frac{\mu}{2}\left(\operatorname{tr} \boldsymbol{b}_{\text {iso }}^{e}-3\right)+\frac{\kappa}{2}\left(\ln \mathbf{J}^{e}\right)^{2} \\
\boldsymbol{\tau}=2 \rho \frac{\partial \psi}{\partial \boldsymbol{b}^{e}} \boldsymbol{b}^{e}=\mu \operatorname{dev}\left(\boldsymbol{b}_{\text {iso }}^{e}\right)+\kappa \ln \mathbf{J}^{e} \mathbf{1}, \quad \operatorname{dev}(\boldsymbol{\tau})=\mu \operatorname{dev}\left(\boldsymbol{b}_{\text {iso }}^{e}\right), \operatorname{vol}(\boldsymbol{\tau})=\kappa \ln \mathbf{J}^{e} \mathbf{1} .
\end{gathered}
$$

Because slip-system tensors are deviatoric by construction, their internal product by the hydrostatic KIRCHHOFF stress components vanishes and the SCHMID stress in (11) remains

$$
\tau^{\alpha}=\mu \overline{\boldsymbol{s}}_{\text {iso }}^{\alpha} \cdot \overline{\boldsymbol{m}}_{\text {iso }}^{\alpha}, \quad \overline{\boldsymbol{s}}_{\text {iso }}^{\alpha}=\boldsymbol{F}_{\text {iso }}^{e} \cdot \overline{\boldsymbol{s}}^{\alpha}, \quad \overline{\boldsymbol{m}}_{\text {iso }}^{\alpha}=\boldsymbol{F}_{\text {iso }}^{e} \cdot \overline{\boldsymbol{m}}^{\alpha} .
$$

\subsection{A rate-dependent formulation via a viscoplastic power-law}

A rate-dependent theory enables the modeling of creep in single crystals and is performed by the introduction of a power law-type constitutive equation for the rates $\dot{\gamma}^{\alpha}$ of inelastic deformation in the slip systems

$$
\dot{\gamma}^{\alpha}=\dot{\gamma}_{0} \frac{\tau^{\alpha}}{\tau_{y}}\left(\frac{\left|\tau^{\alpha}\right|}{\tau_{y}}\right)^{m-1}=\dot{\gamma}_{0} \tau^{\alpha}\left|\tau^{\alpha}\right|^{m-1} \tau_{y}^{-m},
$$

$\dot{\gamma}_{0}$ and $\tau_{y}$ being the reference shear rate and slip resistance, and $m$ being a rate-sensivity parameter. Within an isotropic TAYLOR hardening model, the evolution for the slip resistance $\tau_{y}$ is considered

$$
\dot{\tau}_{y}=\sum_{\alpha} H \cdot\left|\dot{\gamma}^{\alpha}\right|, \quad \gamma=\int_{0}^{t} \dot{\gamma} d t, \quad \dot{\gamma}=\sum_{\alpha} \dot{\gamma}^{\alpha}
$$

\subsection{Incremental kinematics}

The slip rate is discretised with a standard backward EULER integration in order to obtain incremental evolution equations for the update of the evolving quantities

$$
\Delta \gamma^{\alpha}=\Delta t \dot{\gamma}^{\alpha}\left(\boldsymbol{F}^{e}\right)
$$


The implicit exponential integrator is then used to discretise the plastic flow equation (9)

$$
\boldsymbol{F}_{n+1}^{p}=\exp \left[\sum_{\alpha} \Delta \gamma^{\alpha} \overline{\boldsymbol{s}}^{\alpha} \otimes \overline{\boldsymbol{m}}^{\alpha}\right] \cdot \boldsymbol{F}_{n}^{p} .
$$

Due to the property $\operatorname{det}\left[\exp \left(\overline{\boldsymbol{s}}^{\alpha} \otimes \overline{\boldsymbol{m}}^{\alpha}\right)\right]=\exp \left[\operatorname{tr}\left(\overline{\boldsymbol{s}}^{\alpha} \otimes \overline{\boldsymbol{m}}^{\alpha}\right)\right]=\exp (0)=1$, it preserves the plastic volume. Here, $\boldsymbol{F}_{n+1}^{e \text { trial }}=\boldsymbol{f}_{n+1} \boldsymbol{F}_{n}^{e}$, is the trial elastic deformation gradient with $\boldsymbol{f}_{n+1}=\boldsymbol{F}_{n+1} \boldsymbol{F}_{n}^{-1}=\mathbf{1}+\operatorname{grad}_{n}(\Delta \boldsymbol{u})$ and $\mathbf{J}_{n+1}=\operatorname{det} \boldsymbol{F}_{n+1}, \boldsymbol{F}_{\text {iso }}^{\text {trial }}=\mathbf{J}_{n+1}^{-1 / 3} \boldsymbol{F}_{n+1}^{e \text { trial }}$, so that an exponential update for the new elastic deformation gradient can be obtained

$$
\boldsymbol{F}_{n+1}^{e}=\boldsymbol{F}_{n+1}^{e \text { trial }} \cdot \exp \left[\sum_{\alpha}-\Delta \gamma^{\alpha} \overline{\boldsymbol{s}}^{\alpha} \otimes \overline{\boldsymbol{m}}^{\alpha}\right] .
$$

The current trial resolved shear stress $\tau_{n+1}^{\alpha \text { trial }}$, cf. (14), is obtained with the current orientation of the crystal through rotation of the slip system with the trial elastic deformation gradient

$$
\tau_{n+1}^{\alpha \text { trial }}=\mu \overline{\boldsymbol{s}}_{\text {iso }}^{\alpha \text { trial }} \cdot \overline{\boldsymbol{m}}_{\text {iso }}^{\alpha \text { trial }}, \quad \overline{\boldsymbol{s}}_{\text {iso }}^{\alpha \text { trial }}=\boldsymbol{F}_{\text {iso }}^{e \text { trial }} \cdot \overline{\boldsymbol{s}}^{\alpha}, \quad \overline{\boldsymbol{m}}_{\text {iso }}^{\alpha \text { trial }}=\boldsymbol{F}_{\text {iso }}^{e \text { trial }} \cdot \overline{\boldsymbol{m}}^{\alpha} .
$$

\subsection{Equilibrating the plastic state}

Omitting the subscript $n+1$, a residual based on the exponential map is defined to equilibrate the plastic state, leading to a local NEWTON-RAPHSON algorithm through a TAYLOR expansion about the reached point $\boldsymbol{F}_{k}^{e}$

$$
\boldsymbol{R}\left(\boldsymbol{F}^{e}\right):=\boldsymbol{F}^{e}-\boldsymbol{F}^{e \text { trial }} \cdot \exp \left[\sum_{\alpha}-\Delta \gamma^{\alpha} \overline{\boldsymbol{s}}^{\alpha} \otimes \overline{\boldsymbol{m}}^{\alpha}\right]=\mathbf{0}
$$

and

$$
\begin{aligned}
& \boldsymbol{R}_{k}+\partial_{\boldsymbol{F}_{k}^{e}} \boldsymbol{R}\left(\boldsymbol{F}_{k}^{e}\right): \Delta \boldsymbol{F}_{k}^{e}=\mathbf{0} \\
& \Delta \boldsymbol{F}_{k}^{e}=-\left[\partial_{\boldsymbol{F}_{k}^{e}} \boldsymbol{R}\left(\boldsymbol{F}_{k}^{e}\right)\right]^{-1}: \boldsymbol{R}_{k}, \quad \boldsymbol{F}_{k+1}^{e}=\boldsymbol{F}_{k}^{e}+\Delta \boldsymbol{F}_{k}^{e},
\end{aligned}
$$

with the important derivatives

$$
\begin{aligned}
{\left[\partial_{\boldsymbol{F}^{e}} \boldsymbol{R}\left(\boldsymbol{F}^{e}\right)\right]_{i j k l} } & =\delta_{i k} \delta_{j l}+F_{i m}^{e \text { trial }} \mathbb{E}_{m j p q}\left[\sum_{\alpha} \overline{\boldsymbol{s}}^{\alpha} \otimes \overline{\boldsymbol{m}}^{\alpha} \otimes \partial_{\boldsymbol{F}^{e}} \Delta \gamma^{\alpha}\right]_{p q k l} \\
\mathbb{E}_{m j p q} & =\frac{\partial \exp \left(\left[-\sum_{\alpha} \Delta \gamma^{\alpha}\left(\boldsymbol{F}^{e}\right) \overline{\boldsymbol{s}}^{\alpha} \otimes \overline{\boldsymbol{m}}^{\alpha}\right]_{m j}\right)}{\partial\left[-\sum_{\alpha} \Delta \gamma^{\alpha}\left(\boldsymbol{F}^{e}\right) \overline{\boldsymbol{s}}^{\alpha} \otimes \overline{\boldsymbol{m}}^{\alpha}\right]_{p q}},
\end{aligned}
$$

and

$$
\begin{aligned}
\partial_{\boldsymbol{F}^{e}} \Delta \gamma^{\beta} & =\Delta t \dot{\gamma}_{0} m\left|\tau^{\alpha}\right|^{m-1} \tau_{y}^{-m}\left[\Xi^{\alpha \beta}\right]^{-1} \partial_{\boldsymbol{F}^{e}} \tau^{\alpha} \\
\partial_{\boldsymbol{F}^{e}} \tau^{\alpha} & =-\frac{2}{3} \tau^{\alpha} \boldsymbol{F}^{e-T}+\mu \mathbf{J}^{-1 / 3}\left[\overline{\boldsymbol{m}}_{\text {iso }}^{\alpha} \otimes \overline{\boldsymbol{s}}^{\alpha}+\overline{\boldsymbol{s}}_{\text {iso }}^{\alpha} \otimes \overline{\boldsymbol{m}}^{\alpha}\right] \\
\Xi^{\alpha \beta} & =\delta^{\alpha \beta}+\Delta t \dot{\gamma}_{0} m \tau^{\alpha}\left|\tau^{\alpha}\right|^{m-1} \tau_{y}^{-m-1} \sum_{\beta} H \operatorname{sign}\left(\Delta \gamma^{\beta}\right) .
\end{aligned}
$$




\section{GEOMETRICAL MODEL OF THE POLYCRYSTAL}

The polycrystal is modelled with 3D VORONOI cell shaped grains. Through the DELAUNAY triangulation of a given random point seed, a polycrystal of arbitrary size can be obtained through stating the size of the bounding box. For the simulations based on crystal plasticity only, where the nonlinearity is solely due to the constitutive relation of the grain material, the grains are perfectly bonded along their boundaries. In this case, the DELAUNAY refinement algorithm proposed in [21] is used for the discretization of the grain boundaries (facets). Afterwards, the DELAUNAY algorithm proposed in [22] is implemented for the FE discretization of the grain interior, see the result in Fig. 3 .

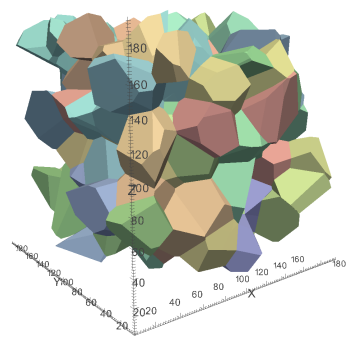

(a) Polycrystal consisting of VORONOI cell grains

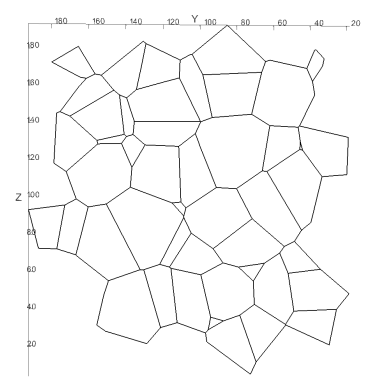

(b) Cut through polcrystalline structure

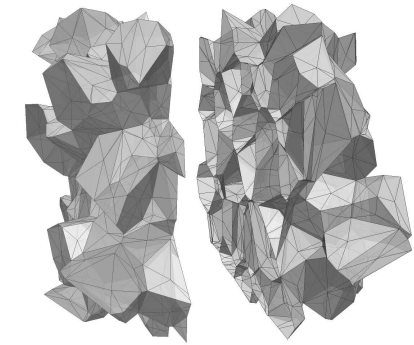

(c) Three-dimensional view into the cutted polycrystal

Figure 3. Polycrystalline model within bounding box $200 \times 200 \times 200 \mu \mathrm{m}$. The VORONOI cell shaped crystal grains are obtained through DELAUNAY triangulation of a random point seed.

In order to realize randomly orientated slip systems in each grain of the undeformed polycrystalline structure, the slip system vectors are rotated around the cartesian axes about three EULER angles $\Phi, \Theta$ and $\Psi$ according to a $y$-convention, see Fig. 4 (first, a rotation about the $z$-axis is performed, then along the $y$-axis and finally along the new $z$-axis)

$$
\begin{aligned}
\boldsymbol{R}_{\Psi} & =\left[\begin{array}{ccc}
\cos \Psi & -\sin \Psi & 0 \\
\sin \Psi & \cos \Psi & 0 \\
0 & 0 & 1
\end{array}\right] \boldsymbol{R}_{\Theta}=\left[\begin{array}{ccc}
\cos \Theta & 0 & \sin \Theta \\
0 & 1 & 0 \\
-\sin \Theta & 0 & \cos \Theta
\end{array}\right] \quad \boldsymbol{R}_{\Phi}=\left[\begin{array}{ccc}
\cos \Phi & -\sin \Phi & 0 \\
\sin \Phi & \cos \Phi & 0 \\
0 & 0 & 1
\end{array}\right] \\
\boldsymbol{R} & =\boldsymbol{R}_{\Psi} \cdot \boldsymbol{R}_{\Theta} \cdot \boldsymbol{R}_{\Phi}
\end{aligned}
$$

Aiming at combining crystal plasticity with cohesive zone models for the simulation of grain boundary sliding and decohesion, zero-thickness interface elements have to be inserted along the grain boundary facets. In $2 \mathrm{D}$, this can be done by duplicating the nodes of the grains along the grain boundaries and constructing the connectivity matrix for the interface elements [5]. In 3D, this procedure is much more complex. The easiest way would be to generate the polycrystalline topology using VORONOI diagrams and then meshing each grain separately from the others. This has the advantage that meshing can be performed by using standard geometry and mesh generation toolkits. However, this procedure may lead to non matching nodes for the construction of the interface elements. Hence, the same procedure as in $2 \mathrm{D}$ is adopted, although it is algorithmically more complex and not possible to be performed 


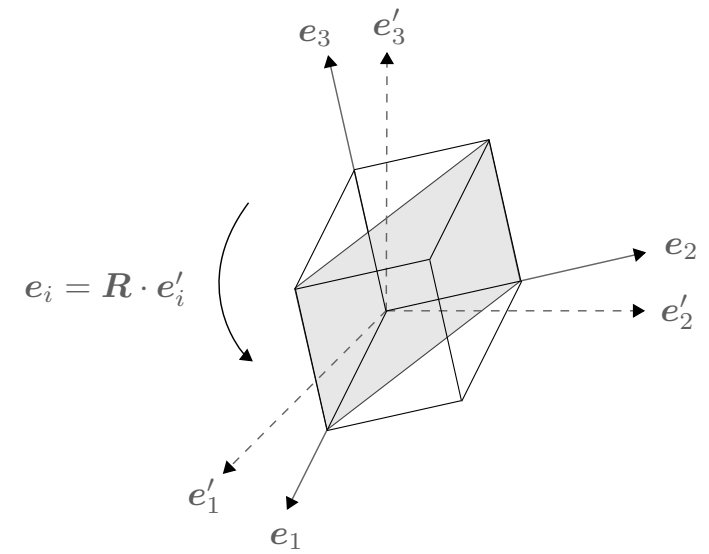

Figure 4. Rotation of the axes around random EULER angles

using commercial mesh generators. Meshing of the grain boundaries and of the grain interior has been done as in case of perfectly bonded grains. Then, node duplication is made for all the nodes pertaining to the grain boundaries, adding them to the existing data structure. The connectivity matrix of the grains is then updated by assigning the new nodes to the finite elements along the grain boundaries. Finally, the connectivity matrix of the interface elements is constructed. The result of this mesh generation procedure is shown in Fig. 5 for a test problem.

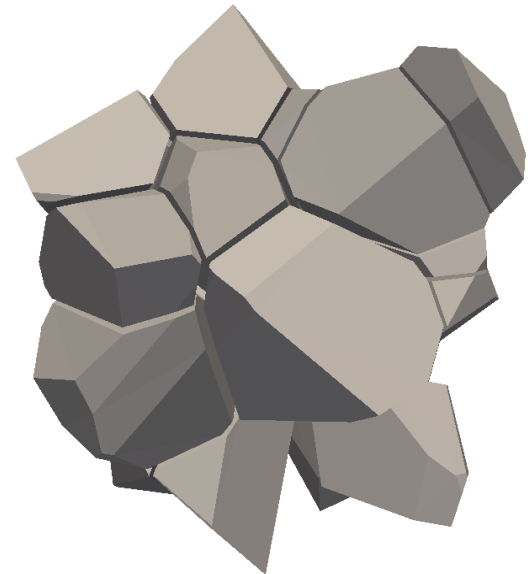

(a) Grains

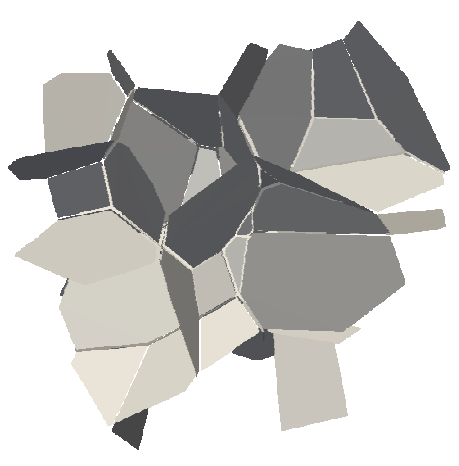

(b) Grain boundaries

Figure 5. 3D grains and grain boundaries of the test problem analyzed in the sequel.

\section{COHESIVE FRACTURE: 3D VS. 2D SIMULATIONS}

In this section, 3D and 2D fracture mechanics responses of a polycrystalline material is investigated. The 3D model has 27 grains and the geometry is shown in Fig. 5. A linear elastic constitutive relation for the grains is adopted, with $E=152200 \mathrm{MPa}$ and $\nu=0.3$. To model grain boundary decohesion, 3D interface elements are inserted along the grain boundaries among the tetrahedra composing the grains. Linear shape functions are considered. The 
constitutive response of the grain boundaries is governed by the CZM presented in Section 2 with a peak traction $\sigma_{\mathrm{p}}=80 \mathrm{MPa}$ and a fracture energy $\mathcal{G}_{F}=0.1 \mathrm{~N} / \mathrm{mm}$ (which is the area under the Mode I traction-separation curve). We also select $l_{\mathrm{Nc}}=l_{\mathrm{Tc}}$ and $\gamma=1$. A tensile test in the $y$-direction is simulated by subdividing the FE nodes on the external rough polycrystalline boundary in three groups. A first group, with fully constrained displacements, includes all the nodes having a $y$-coordinate less than $30 \%$ of the total sample size. A second group with a $y$-coordinate comprised between $30 \%$ and $70 \%$ of the total sample size has free displacements. Finally, the third group with a $y$-coordinate higher than $70 \%$ of the total sample size has an imposed vertical displacement $\delta$. The 2D model is constructed by taking a middle cross-section of the 3D geometry. Similar boundary conditions are imposed to compare with the 3D simulation. The continuum is discretized using quadrilateral elements with linear shape functions and 2D interface elements with linear shape functions are used to discretize the grain boundaries.

The results of the simulations are provided in terms of the total axial force $F$ evaluated at the restrained boundary vs. the imposed axial displacement $\delta$. In general, due to the plane strain assumption of the 2D model, the axial forces of the 2D simulation are much higher than the $3 \mathrm{D}$ ones, since the actual resisting cross-section area in 3D is much smaller than in $2 \mathrm{D}$. To compare results, the forces $F$ and the displacements $\delta$ are divided by the values $F_{y}$ and $\delta_{y}$ corresponding to the end of the linear elastic response of the system. Although the presence of the CZM induces very soon a nonlinearity in the force-displacement curve, the response is not far from linearity for low deformation levels. The comparison in Fig. 6 shows a significant difference between 3D and 2D predictions. The dimensionless peak load is much higher in 2D than in 3D, mostly due to Mixed Mode effects. In 2D, failure is the result of the separation of the lowest grain from the rest of the material microstructure along an interface which is almost perpendicular to the direction of loading, i.e., subjected to Mode I deformation, see Fig. 7. In 3D, the crack pattern at failure involves the separation of several grains separated by inclined interfaces with the occurrence of Mixed Mode deformation, see Fig. 8 .

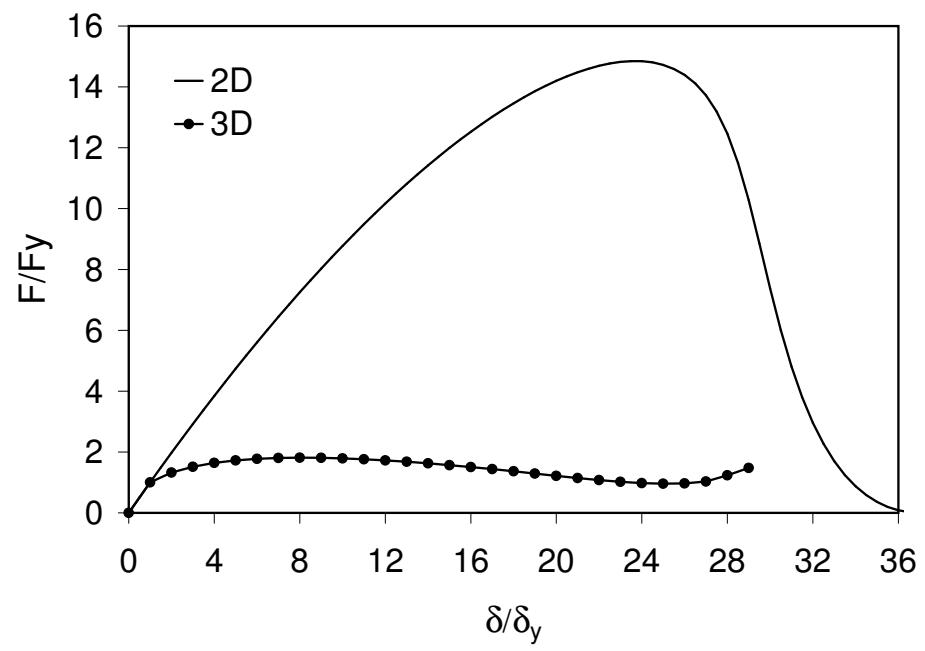

Figure 6. 3D vs. 2D simulation of cracking: dimensionless axial force vs. axial displacement. 


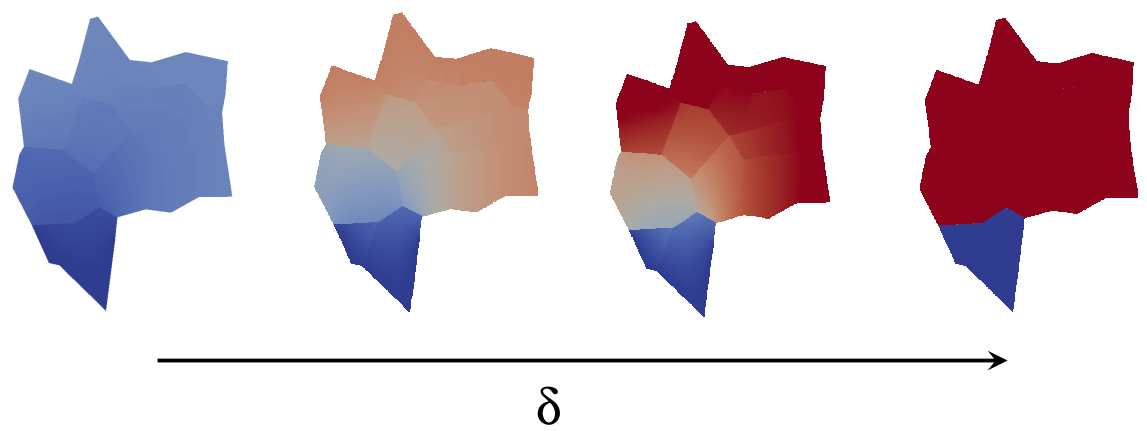

Figure 7. Deformation of the 2D model of the polycrystal, by increasing the imposed boundary displacement $\delta$ until failure. Displacements range from blue (zero displacement) to red (maximum displacement).

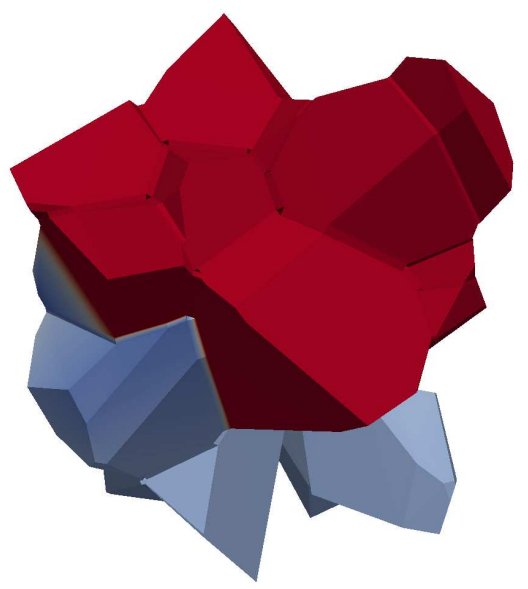

Figure 8. Deformation at failure of the 3D model of the polycrystal. Displacements range from blue (zero displacement) to red (maximum displacement).

\section{PLASTICITY: 3D VS. 2D SIMULATIONS}

In this section, the grains are considered as perfectly bonded and the the nonlinearity is due to the elasto-plastic constitutive law of the grains. In 3D, a crystal plasticity formulation is used, considering 12 slip systems, as appropriate for a face-centered-cubic crystal structure. The slip system vectors are summarized in Tab. 1. The material parameters are $\kappa=152.2$ GPa, $\mu=79.3 \mathrm{GPa}, \tau_{c 0}=80.0 \mathrm{MPa}, \dot{\gamma}_{0}=0.0051 / \mathrm{s}, m=4.0, H=120.0 \mathrm{MPa}$. The geometry of the polycrystal coincides with that analyzed in the previous section. To obtain accurate solutions, the grains are discretized by using tetrahedra elements with quadratic shape functions. As a result, the 3D model has 17021 nodes and 10716 elements.

In the 2D simulation, the same cross-section of the 3D geometry considered in the previous section is considered. The grain boundaries are fully bonded and a VON MISES isotropic plasticity formulation with $\sigma_{y}=80 \mathrm{MPa}$ and absence of hardening is used.

To compare $2 \mathrm{D}$ and $3 \mathrm{D}$ results of the tensile test, the forces and the displacements are made dimensionless by using the corresponding yield values. In the $F / F_{y}$ vs. $\delta / \delta_{y}$ diagram, 


$\begin{array}{cccccccc}\overline{\boldsymbol{m}}^{\alpha} & \overline{\boldsymbol{s}}^{\alpha} & \overline{\boldsymbol{m}}^{\alpha} & \overline{\boldsymbol{s}}^{\alpha} & \overline{\boldsymbol{m}}^{\alpha} & \overline{\boldsymbol{s}}^{\alpha} & \overline{\mathbf{m}}^{\alpha} & \overline{\boldsymbol{s}}^{\alpha} \\ (111) & {[01 \overline{1}]} & (1 \overline{1} \overline{1}) & {[0 \overline{1} 1]} & (\overline{1} 1 \overline{1}) & {[011]} & (\overline{1} \overline{1} 1) & {[0 \overline{1} \overline{1}]} \\ (111) & {[\overline{1} 01]} & (1 \overline{1} \overline{1}) & {[\overline{1} 0 \overline{1}]} & (\overline{1} 1 \overline{1}) & {[10 \overline{1}]} & (\overline{1} \overline{1} 1) & {[101]} \\ (111) & {[1 \overline{1} 0]} & (1 \overline{1} \overline{1}) & {[110]} & (\overline{1} 1 \overline{1}) & {[\overline{1} \overline{1} 0]} & (\overline{1} \overline{1} 1) & {[\overline{1} 10]}\end{array}$

Table 1. 12 fcc slip systems according to [23].

the results do not longer depend on the value of the critical $\tau_{c}$ for the activation of the slip system and on the yield stress $\sigma_{y}$. The difference between 3D and 2D is less pronounced as for the cohesive fracture and mostly regards the progress of the stress saturation. In 3D, the dimensionless force rapidly saturates after yielding, whereas progressive hardening is observed in 2D (Fig. 9). The deformation pattern of the polycrystal is similar in the two cases (see Figs. 10 and 11).

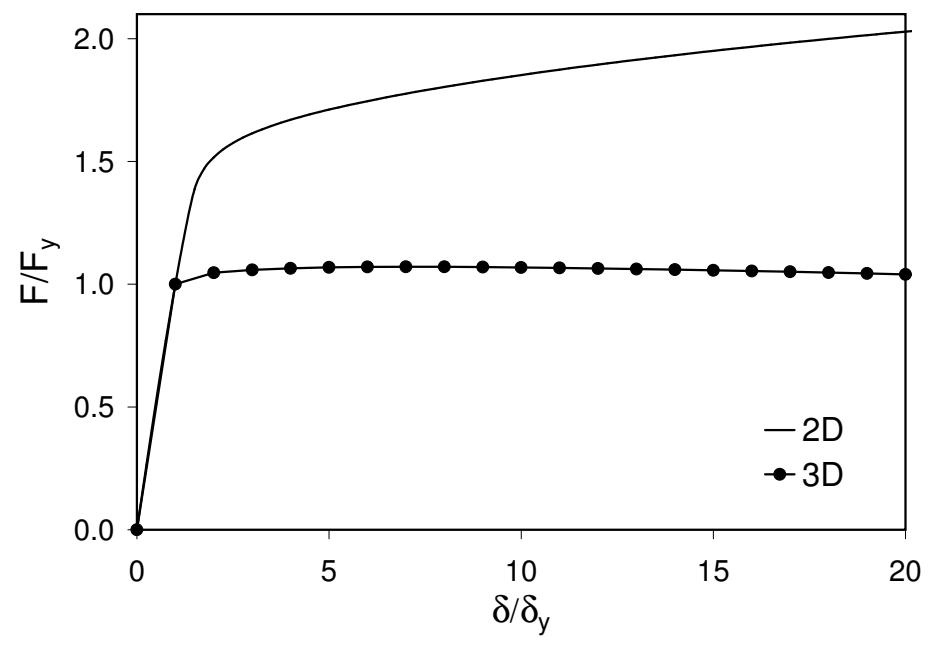

Figure 9. 3D vs. 2D simulation of plastic flow: dimensionless force vs. displacement curves.

\section{INTERPLAY BETWEEN COHESIVE FRACTURE AND PLASTICITY}

To examine the interplay between interface cohesive fracture and grain plasticity, let us consider two blocks joined by a cohesive interface and subjected to a uniaxial tensile test. To this aim, the lower side is restrained to the displacements in the $y$-direction, whereas the upper side is subjected to an imposed displacement $\delta$, see Fig. 12.

Regarding the constitutive models, we consider the blocks as rigid-plastic with hardening, defined by the strain hardening modulus $k_{H}$, see Fig. 13(a). For simplicity, the cohesive zone model (CZM) of the interface is linear with a tension cut-off in correspondence of $\sigma=\sigma_{\mathrm{p}}$. As for linear springs, the CZM is defined by the slope $k_{C}$ in the cohesive traction vs. separation diagram of Fig. 13(b).

During the tensile test, the stress field inside the bulk must be in equilibrium with the cohesive tractions along the interface, as shown in Fig. 12. For each imposed displacement $\delta$, the strain is $\epsilon=\delta / h$, where $h$ is the undeformed vertical size of the sample. Since the bulk 


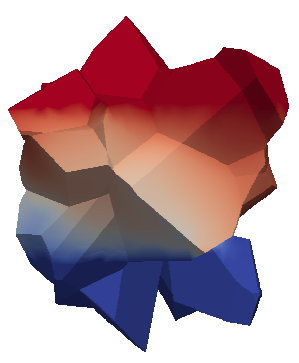

$\delta / \delta_{y}=0.1$

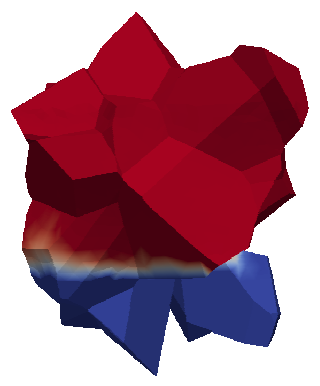

$\delta / \delta_{\mathrm{y}}=1.0$

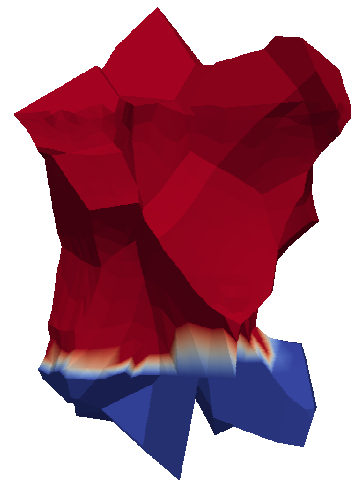

$\delta / \delta_{\mathrm{y}}=20.0$

Figure 10. Cotour plot of the vertical component of the displacement field in the 3D polycrystal and deformed meshes. For each image, the scale ranges from blue (no displacement) to red (maximum displacement).

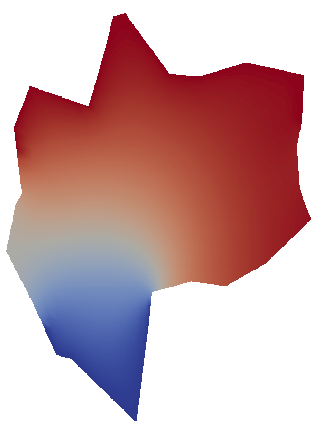

$\delta / \delta_{y}=0.1$

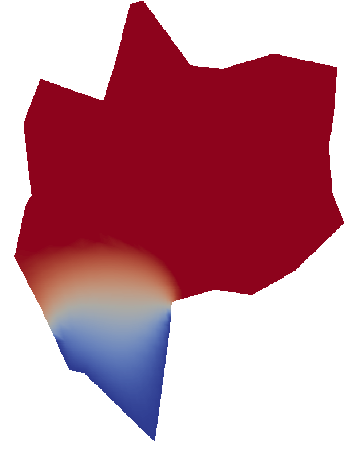

$\delta / \delta_{y}=1.0$

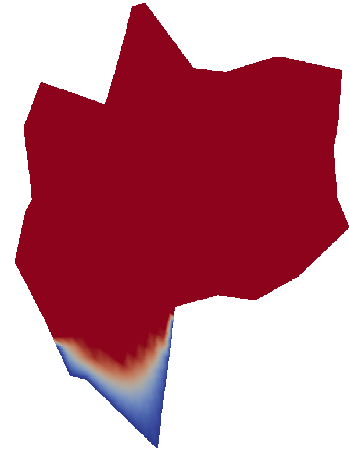

$\delta / \delta_{y}=20.0$

Figure 11. Contour plot of the vertical component of the displacement field in the 2D polycrystal. For each image, the scale ranges from blue (no displacement) to red (maximum displacement).

material is perfectly rigid, the displacement $\delta$ must be totally absorbed by the interface. Hence, the relative opening displacement is $g_{\mathrm{N}}=\delta$. For this separation level, the cohesive traction is equal to $\sigma=k_{C} g_{\mathrm{N}}$. If $\sigma<\sigma_{\mathrm{y}}$, then the representative point $P \equiv(\delta, \sigma)$ of the mechanical response is determined. If, on the other hand, $\sigma_{\mathrm{y}}<\sigma<\sigma_{\mathrm{p}}$, the plastic deformation of the bulk material has to be considered. In this instance, the total specimen deformation is given by:

$$
\delta=\epsilon h+g_{\mathrm{N}},
$$

where $g_{\mathrm{N}}=\sigma / k_{C}$ and $\epsilon$ is related to the stress level by the isotropic hardening equation:

$$
\sigma=\sigma_{\mathrm{y}}+k_{H} \epsilon
$$




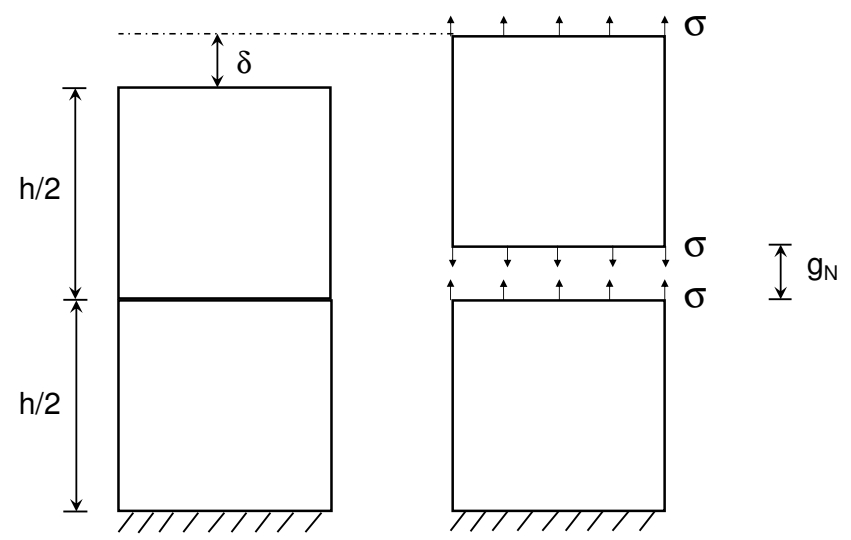

Figure 12. Sketch of the uniaxial tensile test.

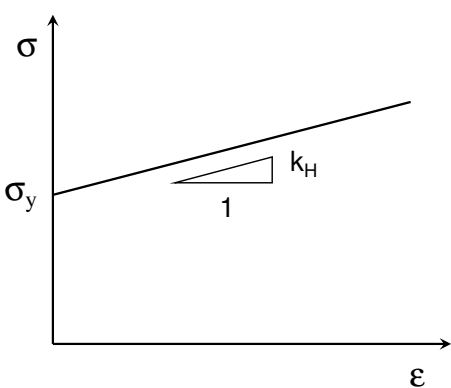

(a)

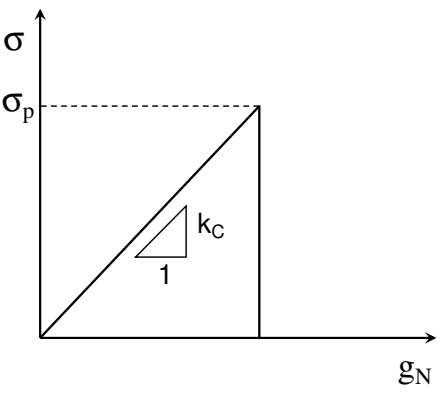

(b)

Figure 13. Constitutive laws: (a) rigid-plastic stress-strain curve for the bulk material and (b) linear CZM with tension cut-off for the interface.

Obtaining $\epsilon$ from Eq.(32) and substituting it in (31), we obtain:

$$
\delta=\frac{\sigma-\sigma_{\mathrm{y}}}{k_{H}} h+\frac{\sigma}{k_{C}}
$$

which gives the unknown stress level for a given axial displacement $\delta$ :

$$
\sigma=\frac{\delta+\sigma_{\mathrm{y}} h / k_{C}}{h / k_{C}+1 / k_{H}}
$$

Finally, for $\sigma>\sigma_{\mathrm{p}}$, the interface cannot sustain tractions and the specimen breaks into two parts.

Although Eq.(34) has been derived under various simplified hypotheses, the following general results can be deduced:

1. The stress-displacement response depends only on the CZM in the range $\sigma<\sigma_{\mathrm{y}}$.

2. A strong interplay between CZM and plasticity takes place for $\sigma>\sigma_{\mathrm{y}}$.

3. The stress at failure is governed by the CZM. 
4. The mechanical response depends on the size $h$ of the sample. For very small specimens $(h \rightarrow 0), \sigma=k_{H} \delta$ and the response is governed by plasticity only. For very large samples, $(h \rightarrow \infty), \sigma=\sigma_{\mathrm{y}} k_{C} / k_{H}$. Since $k_{C} \gg k_{H}$ in practical cases, it is easy to have $k_{C} / k_{H}>\sigma_{\mathrm{p}} / \sigma_{\mathrm{y}}$ and therefore $\sigma>\sigma_{\mathrm{p}}$. Hence, for very large specimens, brittle cohesive failure is expected to dominate over plasticity.

5. In case of perfectly plastic materials $\left(k_{H} \rightarrow 0\right)$, no equilibrium solutions can be found for $\sigma>\sigma_{\mathrm{y}}$.

6. By increasing the ratio $k_{C} / k_{H}$, the mechanical response tends to the rigid-plastic one, see Fig. 14. The limit situation $k_{C} / k_{H} \rightarrow \infty$ corresponds to perfectly bonded interfaces.

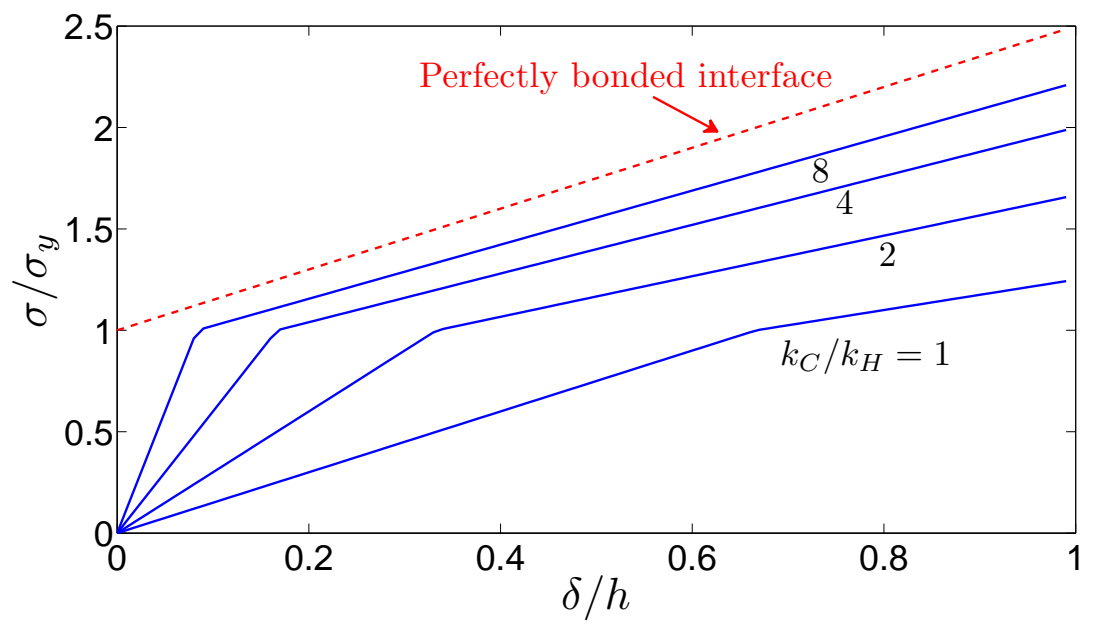

Figure 14. Interplay between CZM and plasticity: rigid-plastic stress-strain constitutive law with hardening for the bulk and linear CZM for the interface.

A more realistic scenario including an elasto-plastic constitutive model for the bulk and a nonlinear softening CZM for the interface leads to a set of equations that cannot be solved in closed form. However, it is possible to proceed numerically by using the normal gap $g_{\mathrm{N}}$ as the driving parameter.

For each value of $g_{\mathrm{N}}$, the cohesive traction $\sigma$, equal to the axial stress in the bulk for equilibrium considerations, is computed according to the CZM relationship. If $\sigma<\sigma_{\mathrm{y}}$, then the strain in the blocks is $\epsilon=\sigma / E$, where $E$ is the Young's modulus of the material. On the other hand, if $\sigma \geq \sigma_{\mathrm{y}}$, the axial strain is computed according to the elasto-plastic constitutive law with hardening, $\epsilon=\sigma_{\mathrm{y}} / E+\left(\sigma-\sigma_{\mathrm{y}}\right) / k_{H}$. In both cases, the total axial displacement is given by $\delta=\epsilon h+g_{\mathrm{N}}$. A particular attention has to be paid to the case when the stress level at a given step $i$ is smaller than the stress at the previous step $(i-1)$. In this instance, due to the stress relief in the bulk, the axial strain has to be computed by considering an unloading along a path parallel to the initial elastic regime.

An example is shown in Fig. 15 by considering an elasto-plastic constitutive model with hardening $\left(E=152000 \mathrm{MPa}, \sigma_{\mathrm{y}}=80 \mathrm{MPa}, k_{H}=120 \mathrm{MPa}\right)$, the nonlinear CZM in [14] with a fracture energy $\mathcal{G}_{F}=0.1 \mathrm{~N} / \mathrm{mm}$ and different values of $\sigma_{\mathrm{p}}$. For $\sigma_{\mathrm{p}}<\sigma_{\mathrm{y}}$, 
plasticity is not activated, the nonlinearity is due to interface decohesion and the mechanical response basically coincides with that of the CZM (see Fig. 15(a)). The elastic contribution of the bulk is negligible for the selected set of material parameters.

For $\sigma_{\mathrm{p}}>\sigma_{\mathrm{y}}$, the response for $\sigma<\sigma_{\mathrm{y}}$ is again due to the combination of the CZM and the elasticity of the system. For higher stresses, plasticity takes place and modifies the final response with respect to the shape of the CZM (see Fig. 15(b)). The interesting aspect is the appearance of a softening response after the hardening branch. For $\sigma_{\mathrm{p}} \gg \sigma_{\mathrm{y}}$, as in Fig. 15(c), the interface strength is very high and the previous trend is amplified.

It is interesting to point out that the numerical results in Fig. 15 are in agreement with the hardening cohesive zone model recently proposed in [24] for metallic materials. In fact, the present curves obtained according to the interplay between CZM and plasticity can be reinterpreted as a new CZM similar to that in [24] by plotting the stresses vs. an axial displacement obtained by removing the elastic component from $\delta$. An example of this procedure is shown in Fig. 16 for the case in Fig. 15(b) (blue solid curve). The initial part of the obtained traction-separation law depends on the shape of the CZM used for the interface. For $\sigma>\sigma_{\mathrm{y}}$, on the other hand, hardening takes place due to plasticity. Finally, an almost linear softening of the cohesive traction is observed for large separations, again due to the CZM formulation.

Increasing the sample size and assuming here that $\sigma_{\mathrm{p}}$ is size-scale independent, the traction-separation law is not scale invariant, although the fracture energy, the peak cohesive traction and the critical opening displacement are the same in all the simulations. In general, the maximum separation corresponding to the onset of linear softening increases with the sample size, again in good agreement with the model proposed in [24].

Similar trends take place when a polycrystal microstructure with several grains and interfaces is considered. For instance, the results of the 2D simulation of the tensile test of the cross-section of the 3D polycrystalline model examined in the previous sections are shown in Fig. 17. The solution in case of a coupled model with VON MISES isotropic plasticity for the grains and the CZM formulation for the interfaces leads to a progressive transition from the CZM solution for small displacements to the elasto-plastic solution for larger displacements. Finally, the post-peak response has again softening as in Fig. 15, this time with a more complex oscillation due to the contribution of several interfaces.

\section{Conclusion}

In this study, two progresses with respect to the state-of-the-art on modelling fracture and plasticity in polycrystalline materials have been made. A first progress regards the setup of a finite element model combining cohesive zone interfaces and crystal plasticity for the simulation of grain boundary decohesion and grain plasticity. Preliminary results show that a strong interplay takes place between these two forms of nonlinearities. As a general trend, the CZM prevails over plasticity for low deformation levels, when the grains are still in their elastic regime. Afterwards, plasticity prevails over CZM. Finally, for a very large deformation level, failure is ruled by the CZM formulation which induces a softening branch. Moreover, the formulation obtained by combining plasticity and CZM is able to capture the well-known size-scale dependencies observed in experimental tests, induced by the interplay between the 


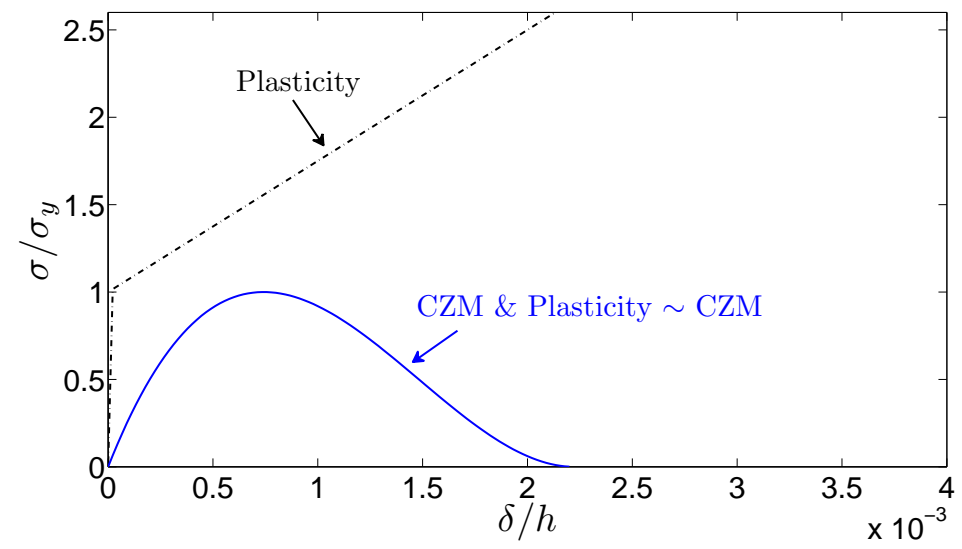

(a) $\sigma_{\mathrm{p}} / \sigma_{\mathrm{y}}=1.0$

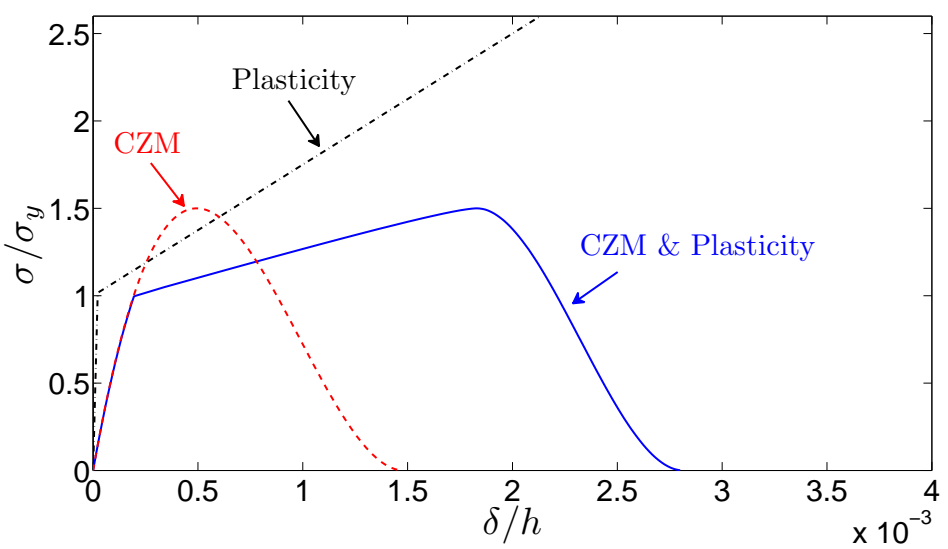

(b) $\sigma_{\mathrm{p}} / \sigma_{\mathrm{y}}=1.5$

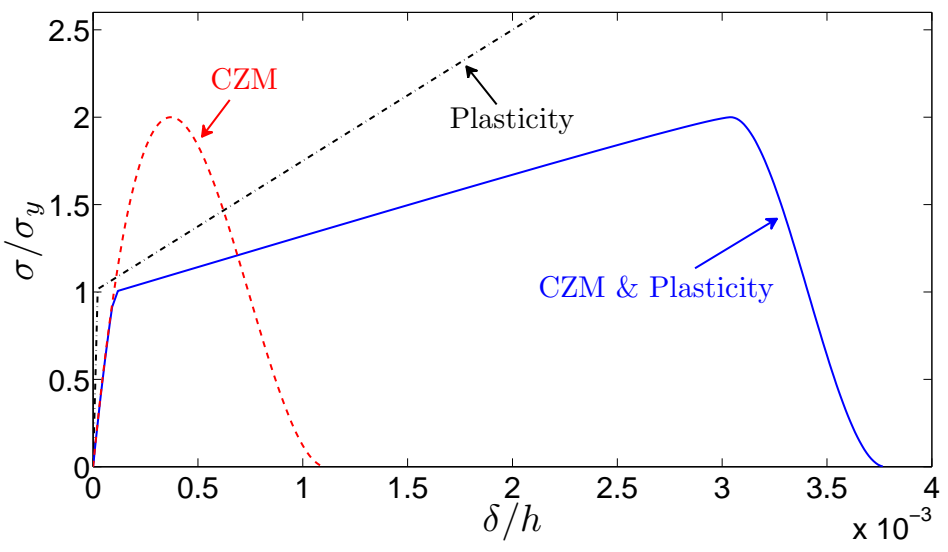

(c) $\sigma_{\mathrm{p}} / \sigma_{\mathrm{y}}=2.0$

Figure 15. Interplay between CZM and plasticity: elasto-plastic with hardening constitutive law and nonlinear CZM.

internal cohesive length and the structural size.

A second progress regards the comparison between 3D and 2D simulations. This has been possible by developing a specific pre-processing to generate $3 \mathrm{D}$ meshes of the polycrys- 


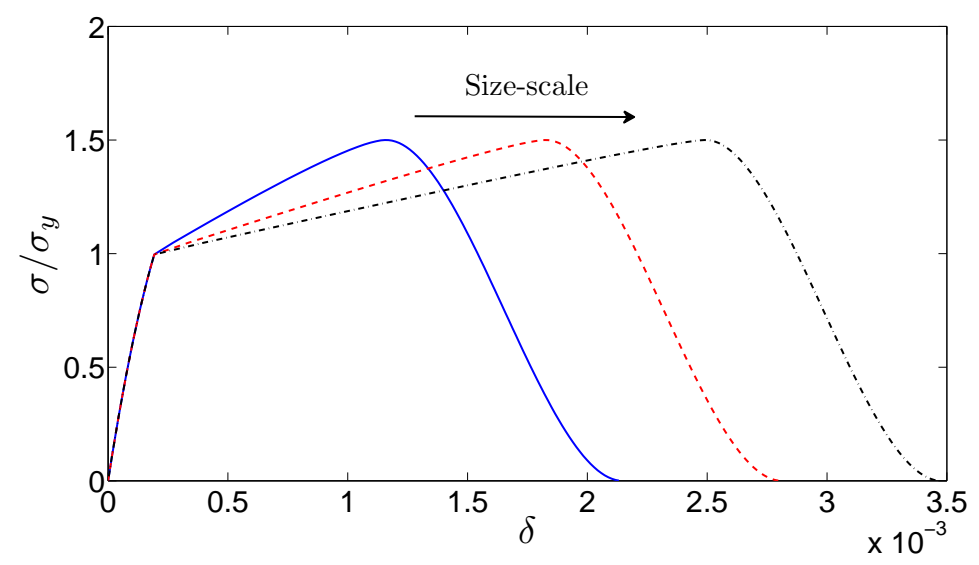

Figure 16. Hardening CZM obtained from the results in Fig. 15(b) and different sizes of the sample.

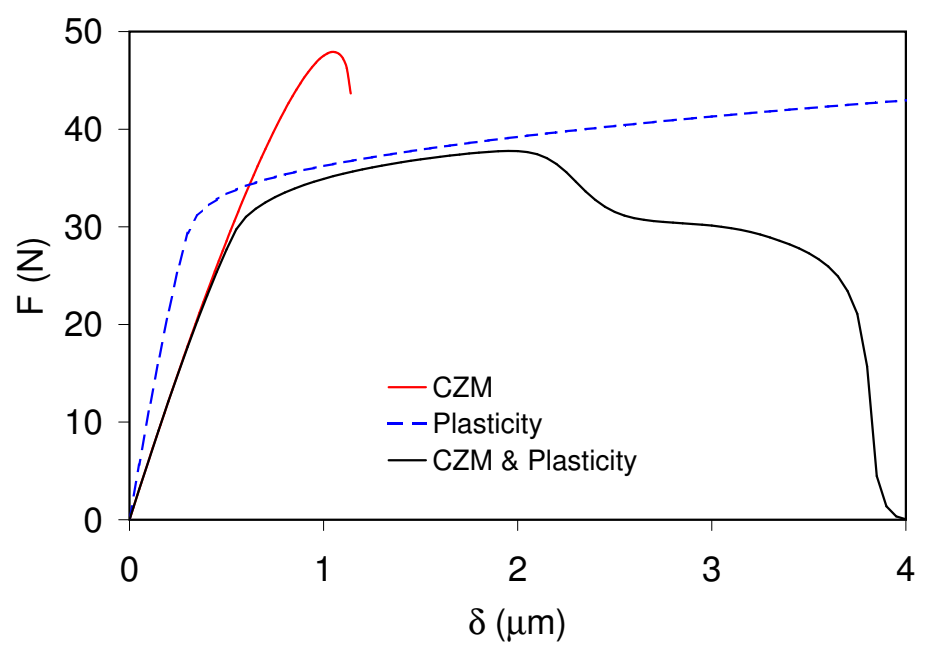

Figure 17. Interplay between CZM and plasticity for the cross-section of the 3D polycrystalline model.

talline grains with interface elements along their internal boundaries. In general, 3D simulations of uniaxial tensile tests differ from the corresponding 2D predictions. The reasons are mainly due to the higher tortuosity of the crack path in 3D and to the fact that the plane strain ansatz does not correspond to the real 3D deformation. The possibility to determine effective mechanical parameters for the $2 \mathrm{D}$ simulations to match the $3 \mathrm{D}$ results is an open issue deserving further investigation.

\section{Acknowledgements}

The support of Ateneo Italo Tedesco (AIT), the Italian Ministry of Education, University and Research (MIUR) and the Deutscher Akademischer Austausch Dienst (DAAD) to the Vigoni project 2011-2012 "3D modelling of crack propagation in polycrystalline materials" is gratefully acknowledged. Financial support for this research was also provided by the Deutsche 
Forschungsgemeinschaft (DFG) under grant SFB TR 73, which is gratefully acknowledged.

\section{References}

[1] H.D Espinosa and P.D. Zavattieri. A grain level model for the study of failure initiation and evolution in polycrystalline brittle materials. part $\mathrm{i}$ : Theory and numerical implementation. Mechanics of Materials, 35:333-364, 2003.

[2] H.D Espinosa and P.D. Zavattieri. A grain level model for the study of failure initiation and evolution in polycrystalline brittle materials. part ii: Numerical examples. Mechanics of Materials, 35:365-394, 2003.

[3] D.H. Warner and J.F. Molinari. A semi-discrete and non-local crystal plasticity model for nanocrystalline metals. Scripta Materialia, 54:1397-1402, 2006.

[4] R.H. Kraft and J.F. Molinari. A statistical investigation of the effects of grain boundary properties on transgranular fracture. Acta Materialia, 56:4739-4749, 2008.

[5] M. Paggi and P. Wriggers. A nonlocal cohesive zone model for finite thickness interfaces - Part II: FE implementation and application to polycrystalline materials. Computational Materials Science, 50:1634-1643, 2011.

[6] Z. Shabir, E. Van der Giessen, C.A. Duarte, and A. Simone. The role of cohesive properties on intergranular crack propagation in brittle polycrystals. Modelling and Simulation in Materials Science and Engineering, 19:035006, 2011.

[7] T. Luther and C. Könke. Polycrystal models for the analysis of intergranular crack growth in metallic materials. Engineering Fracture Mechanics, 76:2332-2343, 2009.

[8] A. Milenin, D.J. Byrska, and O. Grydin. The multi-scale physical and numerical modeling of fracture phenomena in the $\mathrm{MgCa} 0.8$ alloy. Computers \& Structures, 89:10381049, 2011.

[9] O.C. Zienkiewicz and R.L. Taylor. The Finite Element Method. Butterworth-Heinemann, Oxford and Boston, fifth Ed., 2000.

[10] M. Paggi and P. Wriggers. A nonlocal cohesive zone model for finite thickness interfaces - Part I: mathematical formulation and validation with molecular dynamics. Computational Materials Science, 50:1625-1633, 2011.

[11] A. Carpinteri. Cusp catastrophe interpretation of fracture instability. Journal of the Mechanics and Physics of Solids, 37:567-582, 1989.

[12] A. Carpinteri. Softening and snap-back instability in cohesive solids. International Journal for Numerical Methods in Engineering, 28:1521-1537, 1989.

[13] A. Carpinteri. Post-peak and post-bifurcation analysis of cohesive crack propagation. Engineering Fracture Mechanics, 32:265-278, 1989. 
[14] V. Tvergaard. Effect of fiber debonding in a whisker-reinforced metal. Materials Science and Engineering A, 107:23-40, 1990.

[15] J.C.J. Schellekens and R. de Borst. On the numerical integration of interface elements. International Journal for Numerical Methods in Engineering, 36:43-66, 1993.

[16] M. Ortiz and A. Pandolfi. Finite-deformation irreversible cohesive elements for threedimensional crack propagation analysis. International Journal for Numerical Methods in Engineering, 44:1267-1282, 1999.

[17] L. Segurado and J. Llorca. A new three-dimensional interface finite element to simulate fracture in composites. International Journal of Solids and Structures, 41:2977 - 2993, 2004.

[18] D. Peirce, R.J. Asaro, and A. Needleman. An analysis of nonuniform and localized deformation in ductile single crystals. Acta Metallurgica, 30:1087-1119, 1982.

[19] R.J. Asaro and A. Needleman. Texture development and strain hardening in rate dependent polycrystals. Acta Metallurgica, 33:923-953, 1985.

[20] C. Miehe. Exponential map algorithm for stress updates in anisotropic multiplicative elastoplasticity for single crystals. International Journal for Numerical Methods in Engineering, 39:3367-3390, 1996.

[21] J. Ruppert. A Delaunay refinement algorithm for quality 2-dimensional mesh generation. Journal of Algorithms, 18:548-585, 1995.

[22] J. Shewchuk. Tetrahedral mesh generation by Delaunay refinement. In Proc. of the 16th Annu. Sympos. Comput. Geom., 1998.

[23] J.L. Bassani. Plastic Flow of Crystals. In J.W. Hutchinson and T.Y. Wu, editors, Advances in Applied Mechanics, volume 30, pages 191-258. Elsevier, 1994.

[24] A. Carpinteri, B. Gong, and M. Corrado. Hardening cohesive/overlapping zone model for metallic materials: The size-scale independent constitutive law. Engineering Fracture Mechanics, 82:29-45, 2011. 